\title{
Reliability Assessment of LCC Based HVDC Systems Using Public Failure Data
}

\author{
Document Version \\ Submitted manuscript
}

Link to publication record in Manchester Research Explorer

\section{Citation for published version (APA):}

Vilchis-Rodriguez, D., Levi, V., Barnes, M., \& Gupta, R. (2020). Reliability Assessment of LCC Based HVDC Systems Using Public Failure Data. In The 10th International Conference on Power Electronics, Machines and Drives PEMD 2020 (pp. 1-6). [0218] Institution of Engineering and Technology .

\section{Published in:}

The 10th International Conference on Power Electronics, Machines and Drives PEMD 2020

\section{Citing this paper}

Please note that where the full-text provided on Manchester Research Explorer is the Author Accepted Manuscript or Proof version this may differ from the final Published version. If citing, it is advised that you check and use the publisher's definitive version.

\section{General rights}

Copyright and moral rights for the publications made accessible in the Research Explorer are retained by the authors and/or other copyright owners and it is a condition of accessing publications that users recognise and abide by the legal requirements associated with these rights.

\section{Takedown policy}

If you believe that this document breaches copyright please refer to the University of Manchester's Takedown Procedures [http://man.ac.uk/04Y6Bo] or contact uml.scholarlycommunications@manchester.ac.uk providing relevant details, so we can investigate your claim.

\section{OPEN ACCESS}




\title{
RELIABILITY ASSESSMENT OF LCC BASED HVDC SYSTEMS USING PUBLIC FAILURE DATA \\ Damian S. Vilchis-Rodriguez, ${ }^{*}$, Victor Levi ${ }^{1}$, Robin Gupta ${ }^{2}$, Mike Barnes ${ }^{1}$
}

\author{
${ }^{I}$ Department of Electrical and Electronic Engineering, Power \& Energy Division, The University of \\ Manchester, Sackville Street Building, M13 MPL, Manchester, UK \\ ${ }^{2}$ National Grid, National Grid House, Warwick Technology Park, Gallows Hill, CV34 6DA, Warwick, UK \\ *Damian.Vilchis-Rodriguez@manchester.ac.uk
}

Keywords: HVDC, LCC, Reliability Analysis, Data Collating.

\begin{abstract}

\section{Introduction}

A common problem in the reliability analysis of HVDC systems is the lack of representative and sufficient failure data. To alleviate this problem researchers usually rely on public sources of failure data, such as organizations' reports and even data published by other researchers. However, given the inhomogeneous nature of data compiled from various sources, the correctness of the results cannot be verified. In this paper, the adequacy on the use of publicly available failure data to conduct reliability analyses of LCC based HVDC systems is assessed. To this end, failure data available in public domain is first collated. Results from the reliability analysis of two bi-pole topologies of LCC based HVDC systems, with different levels of redundancy and operating voltages, are reported.
\end{abstract}

Lack of representative and sufficient failure data is a common problem faced by researchers in the area of HVDC systems reliability analysis. To alleviate this problem, researchers usually resort to the collection of data from different publicly available sources, such as organizations' reports and from other research articles. However, homogeneity of the information gathered in this way is not guaranteed, given the diverse sources; the generality of any conclusions based on this data is thus affected. In this paper, the adequacy of publicly available failure data for use in reliability analyses of LCC based HVDC systems is assessed. To this end, failure data available in public domain is first collated. Results from the reliability analysis of two bi-pole topologies of LCC based HVDC systems, with different levels of redundancy and operating voltages, are reported.

\section{Data collating}

CIGRE has conduct annual HVDC reliability surveys that currently encompass the period 1968-2016. Biannual reports are published based in the collected information [1-4]. Initially, the reports considered mercury-arc valves and thyristor based systems, however given the diminishing presence of mercury-arc technology, the most recent reports include almost exclusively thyristor systems. It should be noted however that the protocol has been recently revised to collect information related to VSC based HVDC systems [5]. These CIGRE reports can be considered as the most extensive public source of LCC systems reliability data. The reports contain statistical information on energy availability, energy utilization, forced and planned outages and other statistical data that can be used to produce a picture of LCC systems reliability and the relative importance of the system subassemblies.
The extent of the failure data provided in such reports enables the calculation of reliability indices of practical systems under normal operating conditions, as well as the construction of historic data series, which can be used to identify reliability trends. It should be noted that the reliability data contained in the CIGRE reports are confined to failures or events that result in loss of transfer capability. Thus, failures or outages of redundant equipment not resulting in a loss of converter capacity are not reported.

\subsection{Data grouping and reliability indices calculation}

The data on forced outages in the CIGRE reports are classified into seven functional categories, creating a natural subsystem hierarchy. Table 1 lists the equipment categories used in the CIGRE HVDC survey reports [5]. Fig. 1 shows a single-line diagram of a single pole in a typical LCC station [6] where the components that belong to the main categories defined in Table 1 are surrounded with dashed lines for clarity. To facilitate the analysis, the same categories used by the CIGRE are adopted in this paper. This proposed subdivision of the LCC systems in the categories listed in Table 1 is in-line with well-accepted practices. The division of the system into sub-systems is a common method used to facilitate reliability analysis studies. This technique is usually referred to as the "divide and conquer" approach.

Although the subdivision used in this analysis follows a logical and functional relationship between the grouped components, the principal motivation for the use of such categories in this work is the existence of compatible statistical failure data from a reliable source. While convenient to simplify system level analysis, it should be stressed that the lack of granularity on the failure data imposes limitations for the system analysis if component effects need to be investigated. Such limitations will be discussed in more detail below. 
Table 1 CIGRE outages categories and subcategories classification [5]

\begin{tabular}{ll}
\hline Main Category & Sub Category \\
\hline A.C. and Auxiliary & AC Filter and other reactive power Equipment \\
equipment (AC-E) & AC Control and Protection \\
& Converter Transformer (CT) \\
& Synchronous Compensator \\
& Auxiliary Equipment \& Auxiliary Power \\
& Other A.C. Switchyard Equipment \\
& Valve Electrical \\
& Valve Cooling \\
\hline DC Control and & Local Control and Protection \\
Protection Equipment & Master Control and Protection \\
(C-P) & Control and Protection Telecommunications \\
\hline Primary DC Equipment & DC Filters \\
(DC-E) & DC Smoothing Reactor \\
& DC Switching Equipment \\
& DC Ground Electrode \\
& DC Ground Electrode Line \\
& Other DC Switchyard and Valve Hall Equipment \\
\hline DC Transmission Line & Outage events due to Transmission line or \\
(TL) & Cable \\
\hline External AC System & Outage events due to external AC system \\
(EXT) & events \\
\hline Other (O) & Human Error or Unknown \\
\hline
\end{tabular}

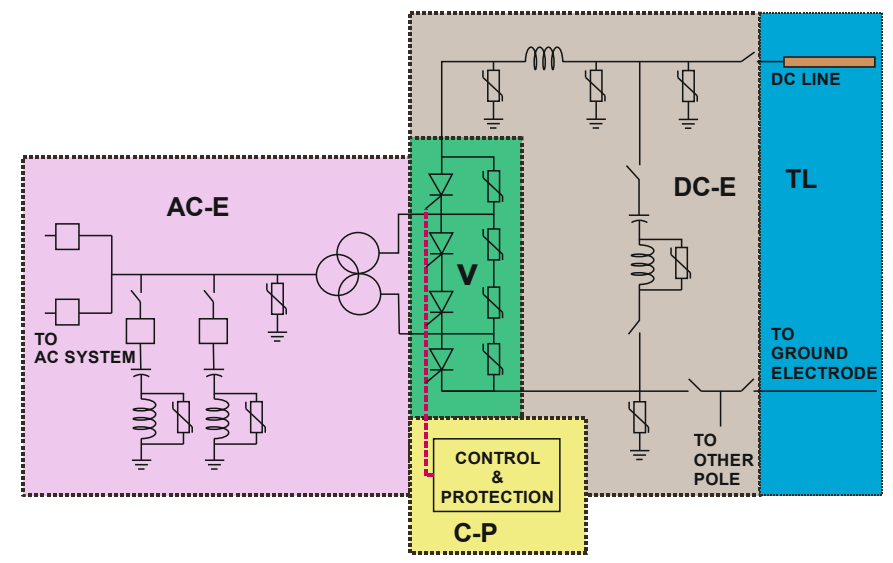

Fig. 1 Typical arrangement of an LCC system, redrawn from [6]

For the analysis, mean time between failures (MTBF) and mean time to repair (MTTR) indices are calculated for all the categories listed in Table 1 using the collected data. The calculated indices are then used in the availability analysis of two typical LCC configurations: conventional bipole system and bipole system with partial converter redundancy. The first topology is typically used in HVDC systems operating up to $500 \mathrm{kV}$, while the latter is commonly used in UHVDC systems. The analyses will be conducted using the Capacity Outage Probability Table (COPT) approach [7].

The reliability of any system is highly dependent on the reliability of the system components. In power systems, the reliability of the components varies considerably depending on voltage and power levels [7]. Therefore, in order to perform realistic reliability analysis of LCC based HVDC links, a practical topology and adequate system ratings must be selected. For reliability analysis, systems and equipment are usually grouped by voltage level-category; one commonly used category is the $300-500 \mathrm{kV}$ [7]. Thus for consistency with existing practices, all the systems included in the CIGRE survey reports for the period 2001 to 2016 with $\mathrm{AC} / \mathrm{DC}$ operating voltages in the $300-500 \mathrm{kV}$ range are considered for the calculation of indices for the conventional topology. Systems above this voltage will be used to derive indices for the topology with partial converter redundancy.

The availability stated in the CIGRE reports is based on the so-called "system energy availability" [5, 6], which is a measure of the amount of energy that could have been transmitted over the HVDC system. In addition, the outage time by category is reported in so-called "equivalent forced outage hours", which is defined by the CIGRE as "the sum of the actual forced outage hours after the outage duration has been adjusted for the percentage of reduction in capacity due to the outage". For example, for one pole outage in a bipole system $(50 \%$ loss of capacity) lasting two hours, the equivalent system outage time would be one hour. When multiple conduction paths exist (e.g. by-pass switches), these must be considered in the calculations of actual outage time.

\subsection{Reliability indices for 300-500 kV system}

For the $300-500 \mathrm{kV}$ system category, the collated data comprise 137 system years from a mix of monopole and bipole installations. From these system years, 38 correspond to bipolar systems (28\%), where only $12.6 \%$ of the reported outages are of bipolar type. Assuming for simplicity that there is a converter at each end of every HVDC pole and that a single DC line is used to link the converters, the actual pole years can be estimated. Thus, 38 additional system years must be considered for the indices calculation to correctly account for the actual number of poles in the data set. Using the two converters/single dc line per pole convention, 350 converters (188 corresponding to 99 monopole system years and 152 from 38 bipole system years) and 175 TLs (99 from the monopole systems and 76 from bipole systems) should be considered. All the associated indices (i.e. C-P, DC-E, TL, etc.) must be adjusted accordingly.

According to the failure data contained in the CIGRE reports for the period 2001-2016, 12\% of the outages in a bipole system were of bipolar nature. Assuming that this percentage is proportionally distributed over the several outage categories, the participation of bipole failures by category can be estimated. Using this criterion and assuming that all the bipole outages were the result of failures on the poles common equipment (i.e. common mode failures) in the C-P, DC-E or TL subsystems (e.g. master control, common dc ground equipment, line-to-line faults), the corresponding common mode failure frequencies can be calculated. Since the two poles are affected when a bipolar outage occurs, the common mode MTTR is assumed to be twice the value of the non-common mode values. Calculated values suitable for the reliability analysis of the 300-500 $\mathrm{kV}$ bipole system are listed in Table 2, where the $\mathrm{cm}$ sub-index refers to common mode failures. 
Table 2 Reliability indices for 300-500 kV AC/DC LCC systems included in CIGRE reports from 2001 to 2016

\begin{tabular}{lccccc}
\hline Category & $\mathbf{f}$ & $\begin{array}{c}\text { MTBF } \\
{[\text { Yrs }]}\end{array}$ & $\begin{array}{c}\text { MTTR } \\
{[\mathrm{hrs}]}\end{array}$ & $\begin{array}{c}\text { Unavail. } \\
{[\mathrm{Hrs} / \mathrm{Yr}]}\end{array}$ & $\begin{array}{c}\text { Unavail. } \\
{[\% / \mathrm{r}]}\end{array}$ \\
\hline AC-E & 0.82 & 0.63 & 8.79 & 7.2078 & 0.0823 \\
\hline $\mathbf{V}$ & 0.36 & 1.45 & 9.41 & 3.3876 & 0.0387 \\
\hline C-P & 0.48 & 0.93 & 4.56 & 2.1888 & 0.0250 \\
\hline DC-E & 0.26 & 1.72 & 14.89 & 3.8714 & 0.0442 \\
\hline $\mathbf{O}$ & 0.21 & 2.38 & 1.88 & 0.3948 & 0.0045 \\
\hline TL & 0.45 & 1.96 & 32.86 & 14.7870 & 0.1688 \\
\hline Tr-failure & 0.10 & 5.00 & 671.29 & 67.1290 & 0.7663 \\
\hline V-damage & 0.01 & 87.5 & 1656.40 & 16.5640 & 0.1891 \\
\hline TL-damage & 0.13 & 7.95 & 891.28 & 115.8664 & 1.3227 \\
\hline C\&P-cm & 0.07 & 7.69 & 9.12 & 0.6384 & 0.0073 \\
\hline DC-E-cm & 0.04 & 14.29 & 29.87 & 1.1948 & 0.0136 \\
\hline TL-cm & 0.06 & 16.67 & 65.72 & 3.9432 & 0.0450 \\
\hline
\end{tabular}

Table $3600 \mathrm{kV}$ (Itaipu system) reliability indices

\begin{tabular}{ccccccc}
\hline Category & $\begin{array}{c}\text { No. } \\
\text { events }\end{array}$ & $\mathbf{f}$ & $\begin{array}{c}\text { MTBF } \\
{[\mathrm{Yrs}]}\end{array}$ & $\begin{array}{c}\text { MTTR } \\
{[\mathrm{hrs}]}\end{array}$ & $\begin{array}{c}\text { Unavai. } \\
{[\mathrm{Hrs} / \mathrm{Yr}]}\end{array}$ & $\begin{array}{c}\text { Unavai. } \\
{[\% / \mathrm{rr}]}\end{array}$ \\
\hline AC-E & $214^{5}$ & 0.2575 & 3.88 & 40.80 & 10.5060 & 0.1199 \\
\hline $\mathbf{V}$ & 73 & 0.0875 & 11.43 & 16.92 & 1.4805 & 0.0169 \\
\hline C-P & 251 & 0.3019 & 3.31 & 5.56 & 1.6786 & 0.0192 \\
\hline DC-E & 79 & 0.0950 & 10.53 & 28.48 & 2.7056 & 0.0309 \\
\hline $\mathbf{O}$ & 205 & 0.2464 & 4.06 & 2.20 & 0.5421 & 0.0062 \\
\hline TL & 84 & 0.4038 & 2.48 & 10.48 & 4.2318 & 0.0483 \\
\hline Tr failure $^{1}$ & 107 & 0.1286 & 7.78 & $72^{4}$ & 9.2592 & 0.1057 \\
\hline V-damage $^{2}$ & 4 & 0.0048 & 208 & 4664 & 22.3872 & 0.2556 \\
\hline
\end{tabular}

${ }^{1}$ Failures reported in [8] between 1970 and 2012 accounting for 52 system years. ${ }^{2}$ Severe faults only. ${ }^{3}$ No cable damage reported only minor TL failures. ${ }^{4} \mathrm{~A}$ three days replacement time is assumed when a spare transformer is available. ${ }^{5}$ Includes CT failures.

Based on the indices listed in Table 2, an average availability of $95.25 \%$ can be calculated. This value is in line with the historic, LCC availability values included in the report. For the period considered, only three installations reported DC cable damage - in all cases submarine cable sections were present. This peculiarity must be considered when the MTTR index is utilized, since the value may not be representative of installations with no submarine sections. Furthermore, the concentration of DC cable failures in only three installations may indicate atypical, localised problems. From the calculated reliability indices, it is clear that physical damage to the converter valves, DC cable or converter transformer results in considerable outage time. However, from the listed values it is also evident that such incidents are rather rare and infrequent.

\subsection{Reliability indices for the $600 \mathrm{kV}$ system}

In contrast with the ample data available for $300-500 \mathrm{kV}$ LCC systems, the Itaipu HVDC system, comprising by two bipole links (bipole 1 and bipole 2), is the only $600 \mathrm{kV} \mathrm{DC}$ link consistently included in the CIGRE reports. Thus the failure data included for the Itaipu system in the CIGRE reports from 1985 (the first time they were included) to 2016 are used for the calculation of the reliability indices for the redundant, $600 \mathrm{kV}$ topology. The reliability indices calculated are listed in Table 3 . The reliability indices in Table 3 reflect the existence of four 12 pulse converters per pole, instead of the two normally present in conventional HVDC bipole configurations. Similarly, four CT groups per pole and associated subsystem (i.e. C-P, DC-E) are considered in the calculations. In line with the actual configuration of the Itaipu system, a single DC line per pole is considered. As can be seen in the table, no severe damage to the DC cable has been reported during the whole 32 year of operating life of the DC link. To attempt to discriminate $\mathrm{CT}$ failures from those related to other components included in the AC-E category, the data used to calculate the CT reliability indices listed in Table 3 were obtained from a set of CIGRE survey reports, whose focus is exclusively on CT reliability [8]. However these reports exhibit limitations, making the data incompatible with those included in the HVDC reports. For instance, the specialized reports cover CT failures only up to the year 2012, and outage times due to CT failures occurred before the year 2003 are not listed in the report. Thus, representative MTTR values for the AC-E category excluding CT failures cannot be established. Therefore, the use of the AC-E indices, which combines CT failures with that of other AC-E components, is preferred for reliability calculations.

Between the years 1985-2016, 29 bipole, 409 pole and 489 converter outages are listed for the Itaipu system in the respective HVDC CIGRE survey reports, which corresponds to $3.13 \%, 44.12 \%$ and $52.75 \%$ of the total number of failures. Compared with the participation of bipole outages calculated for conventional bipole systems $(12 \%)$, the participation of bipole outages for this configuration is substantially lower. In terms of unavailability, bipole failures account only for $0.0036 \%$ of the annual outage time in this installation. This relative low percentage of bipole failures can be attributed to gains on operational flexibility resulting from specific design traits. For instance, considering equipment design and transportation limitations, the design of LCC UHVDC systems adopted the use of two series connected valve-groups in each pole $[9,10]$. High-speed bypass switches are connected to the DC terminal of each valve-group (Fig. 2) to allow bypass the valve-groups that need maintenance or are suffering from component failure or faults [11-13].

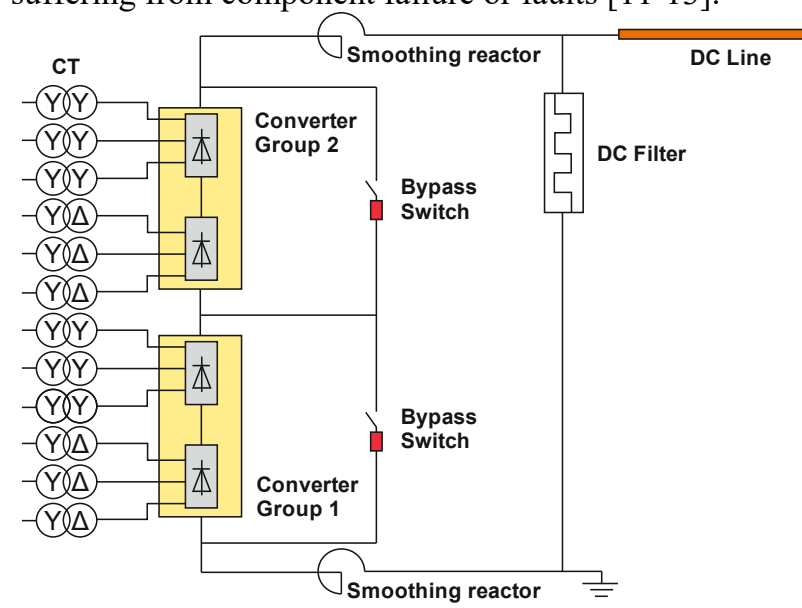

Fig. 2 Converter by-pass switch detail, redrawn from [11] 
This configuration enables additional capacity modes, for example, the mode in which one pole is in half-voltage operation and the other pole is in full-voltage operation. This mode can be achieved by bypassing one valve-group in one pole. The half-voltage pole can keep transmitting power without affecting the full-voltage operating pole. This operating mode will reduce the power loss caused by converter maintenance or component failure. Compared with the conventional one-converter-per-pole configuration, these supplementary operation modes necessarily imply the existence of additional system transfer capacities. Capacity states for the two topologies considered in this paper are discussed in the next section.

\section{Reliability calculations}

As noted in section 2, the aggregate nature of the available failure data impose limitations on the depth of the analysis that can be conducted, mostly due to the impracticability of segregating component specific effects on the overall system reliability, from those induced by other components grouped in the same category. Due to these limitations, simplified representations of the systems are employed in this assessment. The use of analytical models, well suited for the reliability analysis of medium sized systems, was considered sufficient and adequate for the representation of the systems to be analysed. It can be assumed that, depending on system topology, a subsystem failure can only result in one type of failure: monopole (MP), bipole (BP) or partial pole (PP) outage. With this assumption, the different HVDC subsystems can be grouped in categories according to their potential to disrupt the system energy transfer. Fig. 3 shows the reliability logic diagrams for the two HVDC topologies considered in this paper, based on the failure severity effects described before. From the reliability logic diagram in Fig. 3, the combinations necessary to achieve each capacity state can be derived. These are listed in Table 4 and Table 5 for the conventional bipole and the bipole systems with partial converter redundancy, respectively.

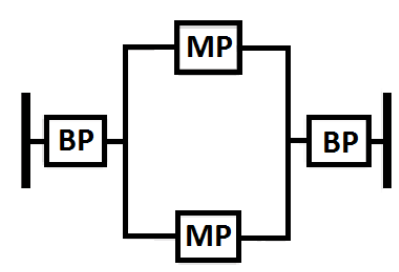

(a)

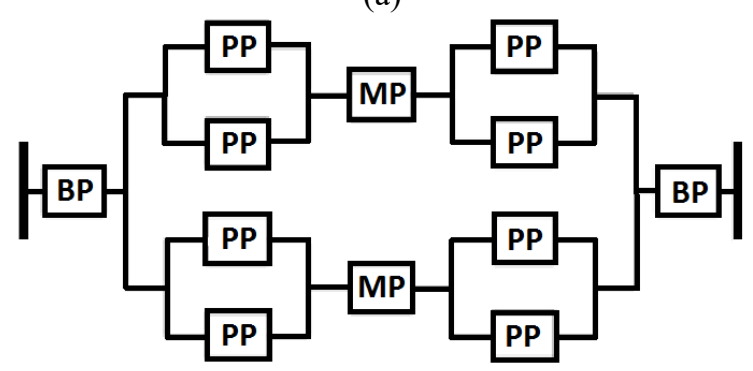

(b)

Fig. 3 Logic diagrams for (a) conventional bipole system and (b) bipole system with partial converter redundancy
Table 4 Power transfer capacity probability for conventional bipolar HVDC system topology

\begin{tabular}{ll}
\hline Capacity [\%] & Failure mode \\
\hline $\mathbf{0}$ & $\begin{array}{l}\text { Any BP outage } \\
\text { A concurrent MP failure in both poles }\end{array}$ \\
\hline $\mathbf{5 0}$ & Any MP failure \\
\hline $\mathbf{1 0 0}$ & Normal operation \\
\hline
\end{tabular}

Table 5 Power transfer capacity probability for bipolar HVDC systems with partial converter redundancy

\begin{tabular}{ll}
\hline Capacity [\%] & Failure mode \\
\hline 0 & Concurrent PP failure in both converters, either in the \\
& rectifier or inverter side, of both poles. \\
& A concurrent PP failure in both converters, either in the \\
& rectifier or inverter side, of one pole and a MP failure in \\
& the other pole. \\
& A concurrent MP failure in both poles. \\
& A BP failure. \\
\hline 25 & A concurrent PP failure in both converters, either in the \\
& rectifier or inverter side, of one pole and any PP failure \\
& in the other pole. \\
& A MP failure in one pole and a concurrent PP failure in \\
& the other pole. \\
\hline $\mathbf{5 0}$ & Any concurrent PP failure in both poles. \\
& Any concurrent PP failure in both converters, either in \\
& the rectifier or inverter side, of one pole. \\
& Any MP failure. \\
\hline $\mathbf{7 5}$ & Any PP failure. \\
\hline & Normal operation
\end{tabular}

For the availability analysis of the non-redundant system, the converter unavailability $\left(\mathrm{Q}_{\text {conv }}\right)$ is assumed to be the result of the summation (reliability of connection in series) of the $\mathrm{V}$, C-P and DC-E subsystems unavailabilities (0.109\%). Once the annual unavailability for each subsystem has been calculated, the probability of occurrence of $0 \%, 50 \%$ and $100 \%$ transfer capacity for the conventional bipole system can be estimated using equations (1) - (3) [14], respectively:

$P_{0}=Q_{M P}^{2}+2 Q_{B P}$

$P_{50}=2 Q_{M P}$

$P_{100}=1-P_{50}-P_{0}$

where:

$Q_{M P}=2 Q_{\text {transf }}+2 Q_{\text {conv }}+2 Q_{A C E}+Q_{T L}$

$Q_{B P}=Q_{C P-c m}+Q_{D C E-c m}+Q_{T L-c m}$

where $Q_{\text {transf }}, Q_{\text {conv }}, Q_{A C E}$ and $Q_{T L}$ are the CT, converter, AC-E and TL subsystem unavailabilities, respectively, and $Q_{C P-c m}$, $Q_{D C E-c m}$ and $Q_{T L-c m}$ are the common mode unavailabilities for the C-P, DC-E and TL subsystems, respectively.

For the $600 \mathrm{kV}$ system, the effect of BP failures is included by assigning to each BP block in Fig. 3b half of the $0.0036 \%$ unavailability resulting from bipole failures. Equations (4)(8) can be used to calculate the probability of occurrence of each capacity state for such system topology: 
$P_{75}=8 Q_{P P}$

$P_{50}=16 Q_{P P}^{2}+4 Q_{P P}^{2}+2 Q_{M P}$

$P_{25}=4 Q_{P P}^{2} 16 Q_{P P}+8 Q_{P P} Q_{M P}$

$P_{0}=16 Q_{P P}{ }^{2} 4 Q_{P P}^{2}+16 Q_{P P}^{2} Q_{M P}+Q_{M P}^{2}+2 Q_{B P}$

$P_{100}=1-P_{75}-P_{50}-P_{25}-P_{0}$

where:

$Q_{c o n v}=Q_{V}+Q_{C P}$

$Q_{P P}=Q_{A C E}+Q_{\text {transf }}+Q_{\text {conv }}$

$Q_{M P}=Q_{D C E}+Q_{T L}$

In eqs. (4)-(8) $P_{100}, P_{75}, P_{50}, P_{25}$ and $P_{0}$ are the probabilities of occurrence of the $100,75,50,25$ and $0 \%$ transfer capacity states, respectively, whilst $Q_{M P}$ and $Q_{P P}$ represent the annual unavailability due to MP and PP outages, respectively, and $Q_{B P}$ is the annual unavailability due to bipole failures. $Q_{V}$, $Q_{C P}$ and $Q_{D C E}$ represent the V, C-P and DC-E subsystems unavailabilities, respectively. Further simplifications of equations (4)-(8) were deliberately avoided in order to show, without ambiguity, how each term in the equations relates to each combination necessary to achieve a specific capacity state. It should be noted that given the difficulties found to segregate $\mathrm{CT}$ failures from those related to other AC-E equipment (caused by the aggregate nature of the CIGRE failure data), the effect of $Q_{\text {transf }}$ in the system reliability was included by using $Q_{A C E}$, which implicitly considers CT failures. Table 6 and Table 7 list the calculated power transfer capacity probability for each possible capacity state for the $300-500 \mathrm{kV}$ and $600 \mathrm{kV}$ system, respectively. Probability values calculated in [16] using failure data for a $500 \mathrm{kV}$ HVDC link, but for a system with a similar topology to that of the $600 \mathrm{kV}$ system used here, are listed in Table 7 for reference. The reliability analysis conducted reveals high energy availability for both configurations. Unsurprisingly, the system with inbuilt redundancy exhibits a higher availability. It is interesting to notice that although sources of failure data and granularity are fundamentally different, the probability values calculated in this assessment are remarkably close to those reported in [11], particularly at full transfer capacity. The results demonstrate that for basic reliability analysis, the use of aggregate failure data, as included in the CIGRE reports, can produce accurate availability estimations and also suggests that the reliability of a $600 \mathrm{kV}$ HVDC system is not significantly different from that of $500 \mathrm{kV}$ system with a similar configuration.

As could be expected, the $75 \%$ power transfer capacity state is the case that most significantly contributes to system unavailability given the relatively large number of blocks that may cause this operating state, as illustrated in Fig. 3b. Furthermore, a sensitivity analysis conducted using equations (4)-(8) revealed that the AC-E subsystem, which is considered part of $\mathrm{Q}_{\mathrm{VG}}$, has the highest impact on the system availability. This is illustrated in Fig. 4, where the influence that each subsystem has on the probability of system operation at $100 \%$ power transfer capacity as the 'normal' failure frequency increases, is shown for each equipment category. In the analysis, the failure frequency values listed in Table 3 are used as 'normal' reference values, with the increase in failure frequency considered separately for each subsystem. As can be seen in the figure, the AC-E subsystem has the highest influence on the system availability. On the other hand, the impact that the other subsystems have on the system availability, for similar failure frequency increases, is in practice rather minor. Increase in failure frequency may be considered to show signs of equipment ageing. However, further analysis revealed that very limited ageing information is contained in the collected failure data.

Table 6 Power transfer capacity probability for $300-500 \mathrm{kV}$ bipolar HVDC systems based in empirical failure data

\begin{tabular}{rr}
\hline Power transfer capacity [\%] & Probability [\%] \\
\hline $\mathbf{0}$ & 0.13 \\
\hline $\mathbf{5 0}$ & 4.16 \\
\hline $\mathbf{1 0 0}$ & 95.71
\end{tabular}

Table 7 Power transfer capacity probability for $600 \mathrm{kV}$ bipolar HVDC systems based on empirical failure data

\begin{tabular}{crr}
\hline Power transfer capacity [\%] & Probability [\%] & Probability [\%] [11] \\
\hline $\mathbf{0}$ & 0.003666 & 0.003100 \\
\hline $\mathbf{2 5}$ & 0.001013 & 0.000600 \\
\hline $\mathbf{5 0}$ & 0.163267 & 0.097400 \\
\hline $\mathbf{7 5}$ & 1.248000 & 1.248100 \\
\hline $\mathbf{1 0 0}$ & 98.584054 & 98.641600 \\
\hline
\end{tabular}

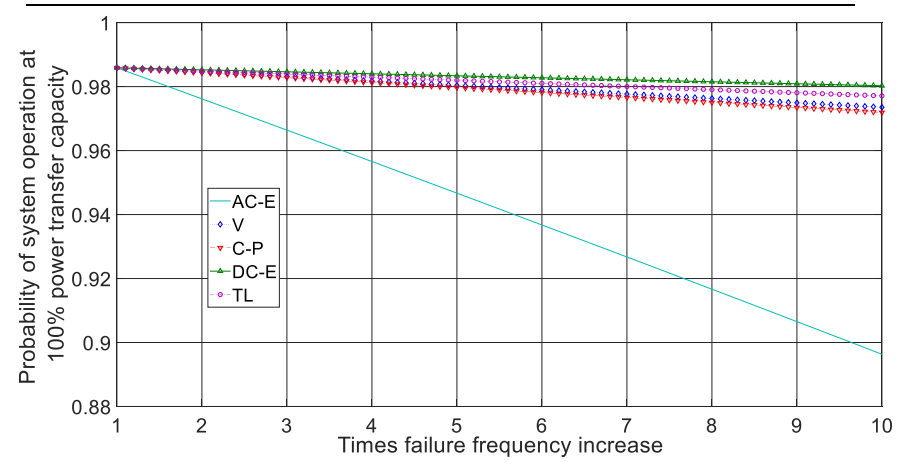

Fig. $4100 \%$ power transfer capacity probability against failure frequency increase

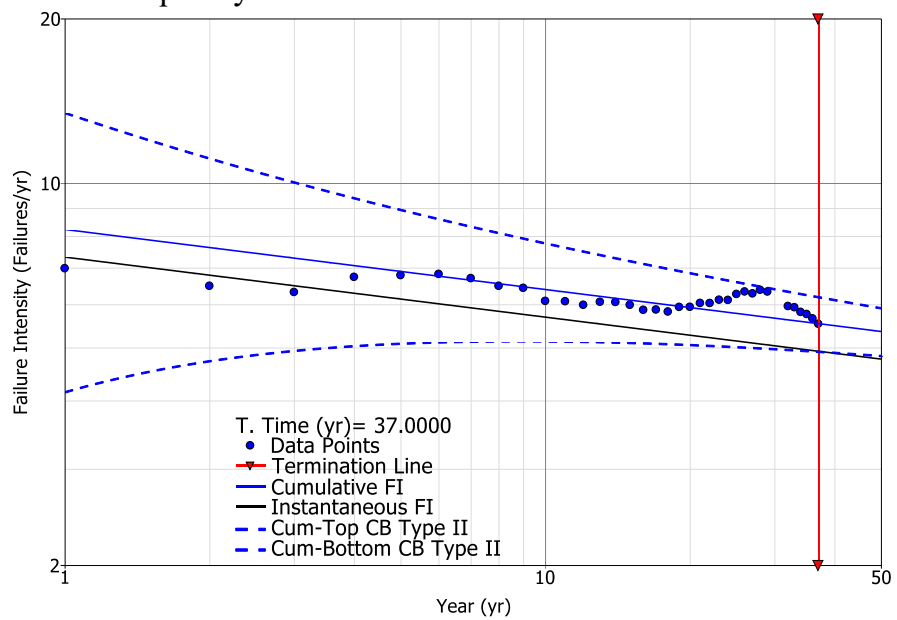

Fig. 5 Failure intensity predicted for all HVDC failures using a Crow-AMSAA (NHPP) model 
Fig. 5 shows results for the failure intensity function obtained using ReliaSoft RGA software assuming a Crow-AMSAA (NHPP) model [15]. Failure intensity can be defined as the number of failures expected in a specified time period. Thus in accordance with the data analysis, instead of increasing, there is a decreasing trend in the failure intensity as the system ages. This result implies that the ageing information is not contained in the data used for the analysis, or is otherwise masked by dominant random failures or current maintenance practices. Similar results were obtained when the failure data for each of the CIGRE categories listed in Table 1 was analysed independently. Individual failure data at a lower level, especially from those components that have been historically linked with poor system unavailability (such as converter transformers, DC cables and converter stations), would be useful to perform more detailed reliability analysis. The availability of failure data at component level is highly desirable to conduct survival analysis required for the design of risk based maintenance management policies. Access to historic failure data at component level is either restricted and not available or of poor quality in the public domain.

\section{Conclusion}

The reliability analysis of LCC based HVDC systems was conducted using failure data available in the public domain. Two bi-pole topologies, with different levels of redundancy and operating voltages, were considered in the analysis. Analytical models suitable for the use with the collected failure data were developed. It was found that the periodic HVDC CIGRE reports contain enough failure data for the elaboration of a basic reliability analysis. However, the aggregation of the failure data by "equipment category" (or subsystem), impairs detailed analysis of the effects that critical system components have on the overall system reliability. The use of failure data by category or subsystem has been found to be adequate for the calculation of representative reliability indices such as MTBT and MTTR. The correctness of the results using the calculated indices was validated by comparison with the published values. Although it is known that voltage level plays a role in system reliability, it was found that published reliability analysis in [11] using data from $500 \mathrm{kV}$ systems resulted in similar availability values to those calculated in this report using failure data from $600 \mathrm{kV}$ systems. This result seems to imply that there is no significant statistical difference between both voltage categories. However, given the very limited failure data available from $600 \mathrm{kV}$ installations, simple coincidence cannot be discarded. More failure data is required to confirm similarity between the two voltage categories.

\section{Acknowledgements}

This work was funded as part of the National Grid/NIA project "The FMEA Studies and Risk-based Maintenance for Emerging Power Electronics Assets within GB Power Networks".

\section{References}

[1] Vancers, I., Christofersen, D.J., Leirbukt, A., et al.: 'A Survey of the Reliability of HVDC Systems Throughout the World During 2001 - 2002,' CIGRE B4-201, 2004.

[2] Vancers, I., Christofersen, D.J., Leirbukt, A., et al.: 'A Survey of the Reliability of HVDC Systems Throughout the World During 2005 - 2006', CIGRE B4-119, 2008.

[3] Bennet, M.G., Dhaliwal, N.S., Leirbukt, A.: 'A Survey of the Reliability of HVDC Systems Throughout the World During 2013 - 2014', CIGRE B4-131, 2016.

[4] Bennet, M.G., Dhaliwal, N.S., Leirbukt, A.: 'A Survey of the Reliability of HVDC Systems Throughout the World During 2015 - 2016', CIGRE B4-137, 2018.

[5] CIGRE WG B4.04: 'Protocol for Reporting the Operational Performance of HVDC Transmission System', CIGRE TB-590, July 2014.

[6] CIGRE WG B4.04: 'Protocol For Reporting Operational Performance of HVDC Systems', CIGRE, Electra, vol. 276, October 1998.

[7] Beddard, A., Barnes, M.: 'Availability analysis of VSCHVDC schemes for offshore windfarms', 6th IET International Conference on Power Electronics, Machines and Drives (PEMD 2012), Bristol, 2012, pp. 1-6.

[8] CIGRE AG B4.04: 'HVDC LCC Converter Transformers, Converter Transformer Failure Survey Results From 2003 To 2012', CIGRE TB-617, April 2015.

[9] Liu, Z., Yu, J., Guo, X., et al.: 'Survey of technologies of line commutated converter based high voltage direct current transmission in China', CSEE J. Power Energy Syst. 2015, 1, $1-8$.

[10] Rao, H., Xu, S., Zhou, Y., et al.: 'Research on Main Circuit Scheme of VSC-UHVDC', Southern Power Syst. Technol. 2017, 11, 1-4.

[11] Xie, K., Hu, B., Singh, C.: 'Reliability Evaluation of Double 12-Pulse Ultra HVDC Transmission Systems', IEEE Trans. Power Del. 2016, 31, 210-218.

[12] Vithayathil, J.J.: 'Bypass operation in bridge convertors for high-voltage d.c. transmission', Proc. IEEE 1965, 112, 359-365.

[13] Machida, T., Yoshida, Y., Fujii, N., et al.: 'Bypass control system for high-voltage dc converter using semiconductor control rectifiers', U.S. Patent 3,636,431, 18 Jan. 1972.

[14] Linden, K., Jacobson, B., Bollen, M.H.J., et al.: 'Reliability study methodology for HVDC grids', CIGRE Sessions, Paris, 2010.

[15] ReliaSoft RGA, https://www.reliasoft.com, last accessed 6th of August 2019. 